\title{
ECONOMIC NETWORKS: WHAT DO WE KNOW AND WHAT DO WE NEED TO KNOW?
}

\author{
FRANK SCHWEITZER* \\ ETH Zurich, D-MTEC, Kreuzplatz 5, \\ 8032 Zurich, Switzerland \\ fschweitzer@ethz.ch \\ GIORGIO FAGIOLO \\ Laboratorio di Economia e Management (LEM), \\ Scuola Superiore Sant'Anna, Piazza Martiri della Liberta 33, \\ 56127 Pisa, Italy \\ DIDIER SORNETTE \\ ETH Zurich, D-MTEC, Kreuzplatz 5, \\ 8032 Zurich, Switzerland \\ and \\ Swiss Finance Institute, c/o University of Geneva, \\ 40 blvd. Du Pont d'Arve, \\ 1211 Geneva 4, Switzerland \\ FERNANDO VEGA-REDONDO \\ Economics Department, European University Institute, \\ Via della Piazzuola 43, 50133 Firenze, Italy \\ and \\ Instituto Valenciano de Investigaciones Economicas, \\ C/Guardia Civil 22 esc. 2 no 1, 46020 Valencia, Spain \\ DOUGLAS R. WHITE \\ Institute of Mathematical Behavioral Sciences, \\ University of California, 3151 Social Science Plaza, \\ Irvine, California 92697, USA
}

Received 2 August 2009

\begin{abstract}
We examine the emergent field of economic networks and explore its ability to shed light on the global and volatile economy where credit, ownership, innovation, investment, and virtually every other economic activity is carried at a scale and scope that respects no geographical, organizational, or political boundaries. In this context, the study of economic networks and their dynamics must reflect the vast complexity of the interaction patterns and integrate it with a realistic account of the incentives and
\end{abstract}

*Corresponding author. 
information that govern agents' behavior. The interplay of both has been shown to produce metastabilities, system crashes, and emergent structures in ways that are yet only poorly understood. Meeting this exciting scientific challenge requires a combination of time-series analysis, complexity theory, and simulation with the analytical tools that have been developed by game theory, as well as graph and matrix theories. We argue that this will help achieving a better integration of theory and data models and provide a better understanding of the potentials and risks of modern economic systems.

Keywords: Economic networks.

\section{Motivation}

The current economic crisis illustrates a critical need for new and fundamental understandings of the structure and dynamics of economic networks. Economic systems are increasingly built on inter-dependencies of both behavior and information, leading to a global economy where credit and investment, trade and input-output flows, research and innovation all occur at a truly world scale that gives rise to a hugely complex system that is difficult to predict and control. Moreover, some inter-dependencies become obvious only during and after the crisis, developing as self-fulfilling phenomena, without precursory signatures.

Specifically, for the current financial crisis, the problems that have initially emerged from thorough analyses include the (lack of) separation of banking and investment, low financial transparency in leveraging, or the breakdown of the necessary trust. In effect, these inherently include factors involving the structure and dynamics of economic networks of all sorts: financial (e.g. reflecting credit or ownership) and otherwise (i.e. "real" ones pertaining to production, trade, and innovation). Their interplay has been argued to lead to an accumulation of excesses in the form of bubbles, whose interaction and mutual reinforcement has led to enormous imbalances explaining the severity of the crisis [62].

The complexity of the modern global economy is exacerbated by the speed and scope at which information flows electronically across national and globally networked markets, with variable intensity of ties and of scale. This makes attempts to understand or control its emergent and volatile networks very difficult indeed. In particular, the danger of cascading failures or the spread of opportunistic behavior through the economic networks is greater today than ever. Self-feeding effects, reinforcing each other through a co-evolving network, can lead to large scale and abrupt consequences that may be too hard to anticipate and tackle.

In sum, the current crisis illustrates the importance and large potential benefits of applying a network approach to the study of the economic system. The frontiers of research examining economic networks have been advancing along two strands: one emanating from economics and sociology, the other from research on complex systems in physics and computer science [55]. In both, nodes represent the different individual actors, or agents, such as firms, banks, or even countries, and links between the nodes describe their mutual interactions, be it trade, ownership, or 
credit/debt relationships. The addition or deletion of either agents or the links between them, and changes in the direction of links, are the fundamentals of network formation.

In a nutshell, our proposed research program is to blend the former two strands of research, adopting from each its strongest points. This entails extending and integrating the traditional paradigm in economic theory (with its emphasis on agents' information and incentives notwithstanding its abstraction from complexity) with the insights and tools developed by the booming field of complex systems (which stresses complexity but ignores those key considerations that underlie agents' behavior). Building on the latter one, statistical physics already made strong attempts to "scale the ivory towers of finance" [19]. However, the emergent field of econophysics $[20,40]$ was met by economists with quite mixed feelings $[15,23]$. Thus, it needs a more substantial approach to merge economic theory with complex systems research. Given the goals of the present discussion we briefly review some of the research in economic networks that is being undertaken along these lines, but also briefly note past impediments to needed inquiry.

\section{The Complex Systems Perspective}

In the complex systems approach, stochastic rules for link formation are tested to find the simplest assumptions that can reproduce statistical regularities in the observed empirical network structure. These rules take into account the characteristic features of the agents $[1,45]$, such as their connectivity degree (number of links attached to that agent/node) or centrality (measuring the importance of a node either through the number of shortest or random paths that pass through it or the recursive weighting of the importance of its neighbors), and do not focus on understanding the endogenous behavior of individual agents as strictly economically motivated agents. These models, therefore, supplement classical economic models to identify the systemic implications of certain network-formation rules on the emerging link structure and of that link structure as a constraint on the options for agents.

At this point in the complex network literature, predictions are often at the aggregate level. Structural properties of networks generated with different stochastic algorithms (e.g. random, scale-free, or small world networks) have been compared with real complex networks, including those in biology (e.g. metabolic and genetic networks), infrastructure (road networks and power grids), communication (internet and mobile phone) and social interaction (e.g. collaborations) [5,8]. The comparison of network structures from these different disciplines suggests that various universality classes can be identified, e.g. based on the distribution of node degrees. For example, in financial contexts, the degree distribution is seen to scale as a power-law for the connections of banks in an inter-bank network $[9,29]$, where the fat tail indicates that there exist few banks interacting with many others. In this example, banks with similar investment behavior form clusters in the network. Similar regularities 
also can be traced for the international trade network (ITN) [17, 18, 24, 49, 56, 57], albeit with some important differences. For instance, the total value of country trade is not power-law distributed, but scales as a log-normal density [18]. This hints to an underlying null model of uncorrelated trade flows, which in turn poses interesting questions about the purported complexity of the ITN.

Other examples are provided by the regional investment or ownership networks $[7,26]$, where European firm-to-firm foreign direct investment (FDI) stock is found to be power-law distributed with the number of employees in the investing firm and in the firm invested in, and with the number of incoming and outgoing investments of both firms. This allows single time-point "predictions" about the investments that regions will receive or make, based on the activity and connectivity of their firms. Thus, firm activity and attractiveness are consonant. Temporal dynamics would need to be studied to see how these variables alter the probability of future activity and attraction in the short and the long run. Data models for networks and the attributes of their nodes and links need to be specified as key elements and relations extrapolated from appropriate raw data to create a correspondence with theoretical variables so that theories can be tested.

Regularities observed on the aggregate level, however, like a degree distribution that follows a power-law, do not imply a specific underlying dynamics of the agents such as preferential attachment [1] to better-connected banks or countries, for example. In other words, this is another example of the unconditional-object problem in empirical validation [10]. Preferential attachment (or proportionate growth) is just one of many generative processes for a power-law distribution [60]. Furthermore, strictly speaking, proportional growth is not sufficient to lead to power-laws. Birth of novel network groups and their possible subsequent demise (death) are two essential ingredients that provide a wealth of regimes and in particular of different power-laws [53]. The universality scaling properties of certain networks, such as power-laws, thus provide only a first-order classification that emphasizes the role of fluctuations and randomness. We predict that the next generation of research will be challenged to measure causality in time series and deviations from universality and allow us to identify the idiosyncratic mechanisms associated with individual agent dynamics and their decision-making processes. This combination should eventually allow us to predict and propose economic policies that favor desired network structures such as those that show themselves more robust to economic shocks. Oversimplification, however, is the casualty of much prior work on universality classes in the topology of networks. Simply put: there has been too much spurious inference from forms of distributions to their generating functions, and without testing through time-series analysis whether these are the actual time-lagged generative processes.

\section{The Socio-Economic Perspective}

In contrast, the socio-economic perspective emphasizes understanding how the strategic behavior of the interacting agents is influenced by — and reciprocally 
shapes — relatively simple changes in network architectures. Economic networks are often viewed through the lens of a network formation game among competing and cooperating agents. In this regard, agents include firms that collaborate in joint R\&D projects [28] or workers who share information on job opportunities [27], and links are added or deleted as the result of purposeful decisions by individual agents that seek to maximize their payoffs. Furthermore, agents must rely on some (generally imperfect and asymmetric) anticipation of what others will do with their (perhaps limited) information about their environment, they frame the problem within some (necessarily bounded) time horizon, and learn from past (and possibly biased) experience of similar situations [22]. These considerations result in a dramatically large number of options (strategies, interactions, etc.) to choose from and therefore agents must be modeled as deciding among them on the basis of boundedly rational rules $[11,50,64]$. Given the lack of empirical evidence for the standard rational agent model, several alternative approaches are currently explored, some of them claiming that agents are even "predictably irrational" with hard-wired biases and quirks [3]. So, there may be a significant component of "noise" or irreproducibility captured by the concept of random utilities [43].

Analyzing economic networks of these sorts involves the use of game theory, which aims at determining the equilibrium (i.e. strategically stable) outcomes, and compares them with what efficiency would require. These problems have been typically addressed within a mathematical framework that is built on stylized simplifications of the situation. In the end, this leads to focusing on the simplest topologies (such as a star or a complete network, where everyone interacts with all others). The task becomes increasingly difficult to solve if the size of the network increases and the topology is allowed to become more complicated.

Nevertheless, the game-theoretic approach is important to highlight the crucial role of incentives in the endogenous and induced behavior of socio-economic networks such as those of collaboration, innovation and $R \& D[4,31,34,35]$. In particular, the competition of interests between individual incentives and aggregate welfare need to be captured, along with their impact on the overall efficiency in the network performance. This tension is well illustrated in the evidence gathered on R\&D collaboration in the innovative human biotech industry [47]. In this disassortative network - the pairing of highly connected and less connected nodes firms in a single multi-connected (cohesive) core also connect with new innovative organizations that are peripheral in the network. Newcomer tie formation in successive years moves them up the cohesive hierarchy composed of successively smaller groups of firms with escalating levels of multi-connectivity. In its first two decades, the network has a metastable 2.5-3.5 year alternation between (a) higher levels of cohesion as newcomers are integrated by core firms that extend their cohesive ties in new areas of research and (b) lower levels of cohesion in periods of high recruitment for novelty. As this study continues while the industry matures, it will be of interest to see if expansion of the cohesive core outstrips recruitment for novelty with the consequence of undermining the disassortivity that has metastabilized the industry. 


\section{Instability and Systemic Risk}

The problem of network formation changes substantially if the underlying environment is subject to persistent volatility, such as rapid innovation, socio-political instability, or environmental change [41] and agents cannot be posited to be at equilibrium [21]. In this context, it is natural to assume that agents follow simple satisficing rules (decision-making strategies that attempt to meet criteria for adequacy, rather than to identify an optimal solution), which they may change in light of their experiences. In such cases, agents cannot hope to attain optimal configurations and, moreover, one finds that the performance of the system can be sharply sensitive to small changes in environmental volatility.

Actually, investigations of complex systems by means of statistical physics [40] have led to the important insight that big disruptions on the system's level do not need large perturbations to occur. Most networks of interest exhibit qualitative changes of regimes in their characteristics and dynamics upon the smooth variations of some "control" parameters or as a function of the network topology and/or metric. These qualitative changes are known under a variety of names, such as ruptures, phase transitions, bifurcations, catastrophes, and tipping points. The ubiquitous tendency to extrapolate new behavior from past ones is fundamentally mistaken at such phase transitions, since the new collective organization is in general completely different from the previous one. Novel models of networks that recognize the role of phase transitions allow us to unify different regimes under a synthetic framework, sometimes with encouraging potential for prediction of crises [59]. Recognizing and using this "phase transition-bifurcation-catastrophe-tipping point" phenomenon is crucial to learn how to diagnose in advance the symptoms of the next great crisis, as most crises occur under only smooth changes of some control variables, without the need for an external shock of large magnitude.

It has been explored in different fields — such as ecosystems or markets how networks that are bipartite or disassortative are thought [42] or shown [29] to lend robustness, within certain limits, against disturbance to the system. For example, the alternation of buyers and suppliers in production chains (avoiding triples and forming hierarchies) also provides structural stability [44]. These types of structures in economic networks, however, have been shown to be vulnerable to cascades of failure: as when production chains lack redundancies, certain ranges of flow parameters lead to insolvencies [6], or problems of pricing created by noncompetitive buyers lead to instabilities [44]. Bankruptcy cascades may occur when suppliers are not paid by those who are their suppliers, or by unexpected shocks to revenues. Studies of local interactions and global network properties go beyond the coupling of global averages [39], as when more firms fail, raising the interest rate for all, causing still more to fail $[6,38]$.

A further level of complexity of disassortative instabilities is shown in the study of an overnight money market [29]. Here, a disassortative network tendency is induced by big lenders having many small borrowers, or the reverse. The dominant 
tendency is metastable (recurrent alternation without a system crash) where reversals depend on whether inter-bank rates toward end-of-month short-term clearing days are decreasing (favoring big lenders) or increasing (favoring buyers). In the loan network this is reflected by changes in the indegree versus outdegree distributions, where the dominant distribution tends to converge at month's end to a power-law. Thus, a macro-feature of the network (lending rates) affects disassortativity and a degree connectivity power-law emerges from the short-term behaviors of the nodes. Metastable dynamical oscillations between these two disassortative states become unstable, however, when overall density of the network of loans passes a critical threshold. As shown by simulation [30] this is because disassortativity is no longer possible and uncertainty becomes greater for both buyers and sellers.

Questions of how standing debts and claims between connected financial institutions affects the probability of a systemic failure has generated interesting insights $[2,37]$. The Lehman Brothers failure offers a real-world example but to provide a predictive theory here requires that we understand longer run dynamics. Most theoretical and empirical methods are not suited to predict cascading network effects. The assumption that a denser network of inter-bank loans or securitization would allow for a better diversification of the failure risk of individual nodes is suspect because risk is only transferred to another level. Simulation studies $[6,38]$ suggest that greater aggregate risk may depend on the coupling strength between nodes. Thus, for financial systems, new measures of systemic risks associated with liquidity and credit exposures will be needed to increase the robustness of the global financial system to idiosyncratic shocks.

Simulations that account for the addition/removal of only single agents to/from the network at each instance of time can produce stable dynamic network models of aggregate risk, but the addition or removal of whole groups of agents to/from the network (e.g. as part of a systemic failure) may result in larger, less predictable effects and drastically change the stability of the system. In this context, it is important to note that networks often fragment and sub-networks coalesce at timescales comparable with those at which epidemics or information spread. We therefore need a unifying conceptual framework [52] to take into account the interplay between these different timescales of grouping, fragmentation, and transmission processes. This allows to reproduce different domain-specific empirical infection profiles, featuring multiple resurgences and abnormal decay times, by simply varying the timescales for group formation and individual transmission [65]. These results emphasize the need to account for the dynamic evolution of multi-connected networks.

In addition, there exist global network effects that are not a priori apparent from the measurable networks of inter-loans, transfer of goods and other exchanges, but result from inter-dependencies in decisions of economic agents. Coming back to the example of Lehman Brothers failure, it was the announcement by the Federal Reserve that the bank will be allowed to go bankrupt, which led to a global 
flight-to-safety and a freeze of inter-bank lending as all other banks realized in a state of shock the potential cascade of defaults that was likely to result. Of course, the bankruptcy of Lehman Brothers played on the underlying real network of bank inter-dependencies, but it also suddenly activated an unrealized channel of attack targeted to the whole system. A similar mechanism explains the surprising cascade of defaults of a small core of Asian countries in 1997. Other countries in the region such as South Korea or Hong Kong had strong economies that were only weakly coupled to the epicenter of the crisis, Thailand. Yet, they were severely affected by the perception of Asian crises. It turned out that this cascade was strongly amplified by a mechanism that is often overlooked, as analysts tend to focus on quantitative data that are blind to it. Specifically, it was the misperception (in behavioral economics, this would be called "framing") by foreign investors that the economies of geographically related countries were linked such that a weakness in one of them was tantamount to a collective illness. The economic basis for this belief was unfounded for South Korea and Hong Kong, yet the geographical framing in the mind of foreign investors made the supposed links between these countries become real: pulling out foreign investments from all these countries simultaneously resulted in a collective and global fight-to-safety; a remarkable example of a selffulfilling prophecy. This mechanism is very similar to bank runs, where just a rumor is in principle sufficient to destroy even the most sound bank if everyone pulls one's money out at the same time. In sum, it is essential to stress and study the existence of networks of inter-dependencies that are not realized yet in the quantitative fluxes or exchanges between nodes. After all, the beliefs of human decision makers are likely to be transmuted into acts, which may aggregate into global impacts, this way suddenly bursting into existence. In consequence, the relevant information on the fragility of a given network requires considering other associated explicit and implicit networks, which need to be understood to avoid future systemic crises.

We note that the dynamics on and of networks can also develop into transient, accelerating, self-reinforcing social bubbles. Examples are collective overenthusiasm as well as unreasonable investments and efforts spreading in coupled networks, which may derive through excessive public and/or political expectations of positive outcomes associated with a general reduction of risk aversion. For example, an analysis of the Apollo program shows [25] that the economic, political, and social factors wove a network of reinforcing feedbacks that led to widespread overenthusiasm and extraordinary commitment by those involved in the project as well as by politicians and by the public at large. The development of such social bubbles seems to be a recurrent dynamical mode appearing when several networks, such as technological, economics, and political, become intertwined into a self-reinforcing spiral.

\section{Beyond Simplicity}

The various examples given above show potential micro-macro-network linkages where local network behavior interacts with more global network structure, i.e. in 
the exchange of knowledge, in trade, or investments. With some simplification, the behavioral or micro-perspective focuses on the system elements, and the global or macro-perspective focuses on the statistical regularities observed at the system level. A key challenge is to identify the paths through which the two largely separate strands of empirical research may converge, given that both graph theory and complexity theory [63] contain ample evidence on the strong theoretical ties between micro-configurations and macro-properties and structures in networks.

In addition to empirical analysis of network structure and dynamical analysis of structural change in networks or the node and link attributes of networks, the field of experimental economics $[12,36]$ provides a source of cross-validation of results to the economic network sciences, and while this cross-fertilization has already begun [47], we predict that these intersections will provide a rich source of stimulation for the next generation of researchers.

The unification of empirical studies on the grounds of basic theoretical commonalities may create a more unified field of economic networks that coalesces in a manner that advances our understanding and leads to further insight and predictions. The theorems of micro-macro-network linkages [35,63] also support closer unification of simulation results and empirical studies, as exemplified here. This advancements should also shed a new light on the problem of heterogeneity.

All economic networks are heterogeneous with respect to both their agents and interaction strength, which can vary in time. Agents may have different preferences, access to resources, failure thresholds, and will not respond to the same influence in the same (predictable) way. Although such variation might be thought to destabilize a system, heterogeneity can also be a source of stability [22], as illustrated, e.g. in decision-making diversity by prediction markets. Moreover, agent features are not constant in time, as they are co-evolving in concert with the network structure and are able to adapt to their environment [32].

Further, network interactions may be multi-level, elements of a given type may be multi-scale, and the types of elements may be multiple, i.e. multi-mode, as with two-mode memberships of agents in organizations. Most models, both in the field of strategic interaction and complex network approaches, ignore these variations.

Moreover, in general, "links" are not just binary (they either exist or not), but are weighted according to the economic interaction under consideration and represent traded volumes, invested capital, etc. and their weight can change over time. Distinguishing networks at different levels of abstraction, e.g. considering directed or undirected, weighted or unweighted links, may illuminate the evolution of their topological properties.

Indeed, findings from study of the ITN [18] emphasize how the topological properties of the network viewed as a binary graph, where only the presence/absence of a trade relation is accounted for, are very different from their weighted counterparts. For example, in the binary ITN, countries with many trade partners typically trade with partners that hold few trade relations, leading to a strongly disassortative network. Conversely, when links are weighted by the value of trade actually flowing through them in a given time interval, say one year, one finds that countries holding 
very strong trade relationships typically trade with many countries, but very intensively with only a few very-connected of them.

Weighting of ties also gives a better sense of how strength of integration in the network differentiates and reflects the patterns of economic growth in different regions. Comparison of high-performing Asian economies (HPAE) with Latin American (LATAM) economies shows very similar trade patterns over the eight five-year periods from 1970 to 2005 when measured by amount of trade or trade relative to GDP, that is, by the aggregate trade attributes of the countries. A country's global centrality in the ITN could be measured by accounting for the likelihood that a given dollar passing from country $\mathrm{X}$ to $\mathrm{Y}$ through links in the network with a probability proportional to their weight (i.e. the value of bilateral trade flowing through the link in a year) passes through country Z, increasing its betweenness score [46]. As described in Refs. 49 and 54, the Asian Tigers have been climbing the ranking of betweenness centrality, whereas LATAM ones remained persistently out of the club of the most central world countries. These results hold when link weights for trade are scaled by importer and/or exporter GDP, i.e. when one washes away country-size supply or demand effects. This removes the correlation of link weights with GDP, but the differentiation between Asian and LATAM countries still holds. Thus, network-based approaches provide a means by which to monitor complex economics systems, and may provide better control in managing and governing these systems.

\section{Novel Ways of Data Analysis}

Knowledge of how systems of connections work will rely on our ability to obtain more and better data, fostering the transition of the field of economics networks from a qualitative to a quantitative and evidence-based science. As computational power increases, it allows large-scale network data on different levels of the economy (e.g. firms, industries, and countries) to be gathered as well as testing of models reflecting the generation of large synthetic data sets. In fact, new means by which business data and internet communication are processed allow for analysis of data soon to be or already available. This includes detailed panel data (longitudinal or cross-sectional time series data) on specific firm interactions (employee flows, R\&D collaborations, etc.) or firm-bank credit market interactions.

The ability to process large data streams will require new tools to squeeze out every last drop of available information reflecting agent interactions and network properties (instead of deriving them from theoretical approaches). Such databases, therefore, may complement both economic network experiments and empirical economic network studies $[33,44,47]$ by allowing large-scale observations in real time $[14,61]$. Studying the relaxation dynamics of a social or economic system after endogenous and exogenous bursts of activity may reveal internal structures of network organization. It also poses the challenge of generalizing in such outof-equilibrium contexts the fluctuation-susceptibility theorem, which has been so 
powerful for accessing the inner properties of complex physical systems at or close to equilibrium [51]. Encouraging results offer a classification of the viral nature of information spreading on social networks [13].

Another very promising line of empirical research and data analysis involves comparing insights obtained by studying the topological properties of different, albeit strongly related, economic networks. A comparison of the topological properties of the ITN and IFN, using the betweenness measure [46] shows that goods markets are more densely connected than financial ones, but both networks display a disassortative, star-shaped structure dominated by a handful of hubs, i.e. very connected countries that in turn interact with weakly disconnected ones. These hubs form a rich club in each network, which are characterized by strong links and can be thought of as the core of each network. This hierarchical structure is more marked in IFN than in the ITN, a feature that can be explained in terms of the existence of economies of scale and scope in the processing of information inherent to financial intermediation. Economies at such scale lead to the emergence of large financial centers that offer a more efficient intermediation and therefore attract many partners. Moreover, their high-income countries tend to be more integrated and more clustered [54]. Hence, they act as hubs for poorer economies, so that a hierarchy exists also in terms of economic development.

Economic networks, as other real-world systems, also evolve in physical space as well as time. The transmission of information or the adoption of a new states and physical distances for interaction, such as trade, occurs over natural timescales. This challenges both theoretical concepts and use of raw data for empirical validation purposes. Borrowing from theoretical and applied work in social sciences, one might map real-world constraints of time and space in manners that may be advantageous to scientific advances.

Time-dependent resolution of the properties of economic networks moves beyond a single-snapshot approach, and allows the researcher to identify conditions for dynamical or path-dependent evolution of networks by combining findings with complementary information, i.e. the correlations between economic network evolution and other macro-economic dynamics. Most previous studies of networks are based on the assumption that networks are more permanent than they really are. For example, the longitudinal analysis of human biotechnology [47] suggests that there is a life cycle of research and development networks related to the timing of the exchange of knowledge. As pointed out above, explaining the existence of many coexisting timescales associated with the evolution of networks and their relevance in the dynamics occurring on them is crucial.

Extracting network structure from reported data, in particular for aggregated economic data, is very difficult. For example, the banking sector does not make all debt/credit relationships publicly available although theoretical decompositions of aggregated data have been studied [9]. Even then, analyses may resemble reading tea leaves: only what was previously known or predicted is revealed. Statistical regularities in economic networks have been identified through sheer data processing, 
but challenges the importance of the various measures that are input in large-scale network characterization. Thus, the utility of each measure needs to be critically examined.

Specifically, information about the role of agents and their function or their influence in an evolving economic network needs to be extracted $[48,58]$. Given that measures such as multi-connective cohesion are useful indicators [47] related to causal processes, one might handle their computational complexity through cloud computing and use of supercomputers, or look to matrix-based methods that focus on cycle density in networks such as sub-group centrality [16].

New methods are needed to identify patterns and new concepts to quantify control (direct and indirect) need to be developed. Promising steps have already been taken, as demonstrated by the identification of the backbone of control in ownership networks [26] and roles defined by structural position [48] or centrality rankings [49] in the ITN.

\section{Conclusions}

The network approach brings a whole new perspective on the role of co-evolving inter-dependency in large and complex economic systems. We anticipate that a new wave of research should begin to merge the description of individual agent's strategies with their co-evolving networks of interactions, in ways that are enriched by insights discovered from simulation [35]. There is much more to discover from approaches combining the economic emphasis of individual strategic decisions with a network approach of interactions and adaptive feedbacks. Sometimes, such methods may lead to single equilibria, but more often they result in multiple coexisting equilibria, regime shifts and out-of-equilibrium transients, as well as sudden bifurcations to new regimes which more accurately characterize real-world systems.

In this way, we should obtain new perspectives on the principles that make economic networks robust and efficient in the face of network complexity. Causal analysis of time series will be needed if better policies, e.g. both to reduce conflicts between individual interests and the risk of global failure, can be designed. Network simulations of the dynamics of innovation involving transfer and growth of knowledge shows that network formation is inefficient if the time to evaluate new links is too short $[34,35]$, which matches findings about time-lags for assimilating new knowledge and innovation in knowledge industries [47].

A rich research agenda in economic networks is being built upon the foundation of self-organization resulting from the interplay between agents' decision making and the dynamic interactions among them. However, we argue that to maximize the information from such studies three complementary lines of research must be pursued: (a) empirical studies providing insights into economic networks from massive data analysis, (b) theory encompassing the appropriate description of economic agents (heterogeneity, strategic interaction) and their interactions (network dynamics, time boundedness, co-evolution of agents and interactions), and (c) a systemic 
perspective bestowing a new understanding of systemic effects as coming from varying network interactions.

\section{Acknowledgments}

F.S. and D.S. acknowledge financial support from the ETH Competence Center "Coping with Crises in Complex Socio-Economic Systems" (CCSS) through ETH Research Grant CH1-01-08-2. F.V-R. gratefully acknowledges financial support from the Spanish Ministry of Education under grant SEJ2007-62656. D.W. work is supported by external faculty funding at the Santa Fe Institute and anonymous nonprofit contributions to the UC Irvine faculty group in Social Dynamics and Complexity.

\section{References}

[1] Albert, R. and Barabási, A.-L., Statistical mechanics of complex networks, Rev. Mod. Phys. 74 (2002) 47-97.

[2] Allen, F. and Gale, D., Financial contagion, J. Polit. Econ. 108 (2000) 1-33.

[3] Ariely, D., Predictably Irrational: The Hidden Forces That Shape Our Decisions (Harper Collins, New York, 2008).

[4] Bala, V. and Goyal, S., A noncooperative model of network formation, Econometrica 68 (2000) 1181-1230.

[5] Barrat, A., Barthelemy, M. and Vespignani, A., Dynamical Processes on Complex Networks (Cambridge University Press, 2008).

[6] Battiston, S., Delli Gatti, D., Gallegati, M., Greenwald, B. and Stiglitz, J., Credit chains and bankruptcy propagation in production networks, J. Econ. Dyn. Cont. 31 (2007) 2061-2084.

[7] Battiston, S., Rodrigues, J. F. and Zeytinoglu, H., The network of inter-regional direct investment stocks across Europe, Adv. Complex Syst. 10 (2007) 29-51.

[8] Borgatti, S., Mehra, A., Brass, D. and Labianca, G., Network analysis in the social sciences, Science 323 (2009) 892.

[9] Boss, M., Elsinger, H., Summer, M. and Thurner, S., Network topology of the interbank market, Quant. Finance 4 (2004) 677-684.

[10] Brock, W., Scaling in economics: A reader's guide, Indus. Corp. Change 8 (1999) 409-446.

[11] Brock, W. and Durlauf, S., Discrete choice with social interactions, Rev. Econ. Studies 68 (2001) 235-260.

[12] Callander, S. and Plott, C., Principles of network development and evolution: An experimental study, J. Public Econ. 89 (2005) 1469-1495.

[13] Crane, R. and Sornette, D., Robust dynamic classes revealed by measuring the response function of a social system, Proc. Nat. Acad. Sci. USA 105 (2008) 1564915653.

[14] Deschatres, F. and Sornette, D., The dynamics of book sales: Endogenous versus exogenous shocks in complex networks, Phys. Rev. E 72 (2005) 016112.

[15] Di Matteo, T. and Aste, T., No worries: Trends in econophysics, Eur. Phys. J. B 55 (2007) 121-122.

[16] Estrada, E. and Rodriguez, J. A., Subgraph centrality in complex networks, Phys. Rev. E 71 (2005) 056103. 
[17] Fagiolo, G., Reyes, J. and Schiavo, S., On the topological properties of the world trade web: A weighted network analysis, Phys. A 387 (2008) 3868-3873.

[18] Fagiolo, G., Schiavo, S. and Reyes, J., World-trade web: Topological properties, dynamics, and evolution, Phys. Rev. E 79 (2009) 036115.

[19] Farmer, J., Physicists attempt to scale the ivory towers of finance, Comput. Sci. Eng. 1 (1999) 26-39.

[20] Farmer, J., Shubik, M. and Smith, E., Is economics the next physical science? Phys. Today 58 (2005) 37-42.

[21] Farmer, J. D. and Geanakoplos, J., The virtues and vices of equilibrium and the future of financial economics, Complexity 14 (2009) 11-38.

[22] Galeotti, A., Goyal, S. and Kamphorst, J., Network formation with heterogeneous players, Games Econ. Behav. 54 (2006) 353-372.

[23] Gallegati, M., Keen, S., Lux, T. and Ormerod, P., Worrying trends in econophysics, Physica A $\mathbf{3 7 0}$ (2006) 1.

[24] Garlaschelli, D. and Loffredo, M., Structure and evolution of the world trade network, Physica A 355 (2005) 138-144.

[25] Gisler, M. and Sornette, D., Exuberant innovations: The Apollo program, Society 46 (2009) 55-68.

[26] Glattfelder, J. and Battiston, S., The backbone of complex networks of corporations: Who is controlling whom? (2009), http://arxiv.org/abs/0902.0878.

[27] Granovetter, M., Getting a Job: A Study of Contacts and Careers (University of Chicago Press, 1995).

[28] Hagedoorn, J., Inter-firm R\&D partnerships: An overview of major trends and patterns since 1960, Res. Policy 31 (2002) 477-492.

[29] Iori, G., De Masi, G., Precup, O., Gabbi, G. and Caldarelli, G., A network analysis of the Italian overnight money market, J. Econ. Dyn. Cont. 32 (2008) 259-278.

[30] Iori, G. and Jafarey, S., Criticality in a model of banking crises, Phys. A 299 (2001) 201-221.

[31] Jackson, M., Social and Economic Networks (Princeton University Press, 2008).

[32] Kirman, A., The economy as an evolving network, J. Evol. Econ. 7 (1997) 339-353.

[33] Kogut, B. and Walker, G., The small world of Germany and the durability of national networks, Am. Sociol. Rev. 66 (2001) 317-335.

[34] König, M. D., Battiston, S. and Frank, S., Modeling evolving innovation networks, in Innovation Networks: New Approaches in Modeling and Analyzing, eds. Pyka, A. and Scharnhorst, A. (Springer, Heidelberg, 2009), pp. 187-267.

[35] König, M. D., Battiston, S., Napoletano, M. and Schweitzer, F., On algebraic graph theory and the dynamics of innovation networks, Netw. Heterogen. Media 3 (2008) 201-219.

[36] Kosfeld, M., Economic networks in the laboratory: A survey, Rev. Netw. Econ. 3 (2004) 20-41.

[37] Leitner, Y., Financial networks: Contagion, commitment, and private sector bailouts, J. Finance 60 (2005) 2925-2953.

[38] Lorenz, J. and Battiston, S., Systemic risk in a network fragility model analyzed with probability density evolution of persistent random walks, Netw. Heterogen. Media 3 (2008) 185-200.

[39] Lorenz, J., Battiston, S. and Schweitzer, F., Systemic risk in a unifying framework for cascading processes on networks (2009), http://arxiv.org/abs/0907.5325, Eur. Phys. J. $B$ (forthcoming).

[40] Mantegna, H. E. and Stanley, R. N., An Introduction to Econophysics (Cambridge University Press, 2000). 
[41] Marsili, M., Vega-Redondo, F. and Slanina, F., The rise and fall of a networked society: A formal model, Proc. Nat. Acad. Sci. 101 (2004) 1439-1442.

[42] May, R., Levin, S. and Sugihara, G., Ecology for bankers, Nature 451 (2008) 893-895.

[43] McFadden, D., Econometric analysis of qualitative response models, in Handbook of Econometrics, eds. Griliches, Z. and Intrilligator, M., Vol. II (Elsevier, Amsterdam, 1974).

[44] Nakano, T. and White, D., Network structures in industrial pricing: The effect of emergent roles in Tokyo supplier-chain hierarchies, Struct. Dyn.: e-J. Anthropol. Relat. Sci. 2 (2007) 1.

[45] Newman, M., The structure and function of complex networks, SIAM Rev. 45 (2003) $167-256$.

[46] Newman, M., A measure of betweenness centrality based on random walks, Soc. Netw. 27 (2005) 39-54.

[47] Powell, W., White, D., Koput, K. and Owen-Smith, J., Network dynamics and field evolution: The growth of interorganizational collaboration in the life sciences, $\mathrm{Am}$. J. Sociol. 110 (2005) 1132-1205.

[48] Reichardt, J. and White, D., Role models for complex networks, Eur. Phys. J. BCondens. Matter Complex Syst. 60 (2007) 217-224.

[49] Reyes, J., Schiavo, S. and Fagiolo, G., Assessing the evolution of international economic integration using random-walk betweenness centrality: The cases of East Asia and Latin America, Adv. Complex Syst. 11 (2008) 685-702.

[50] Rubinstein, A., Modelling Bounded Rationality (MIT Press, 1998).

[51] Ruelle, D., Conversations on nonequilibrium physics with an extraterrestrial, Phys. Today 57 (2004) 48-53.

[52] Ruszczycki, B., Burnett, B., Zhao, Z. and Johnson, N., Relating the microscopic rules in coalescence-fragmentation models to the macroscopic cluster size distributions which emerge (2008), http://arxiv.org/abs/0808.0032.

[53] Saichev, A., Malevergne, Y. and Sornette, D., Theory of Zipf's Law and Beyond, Lecture Notes in Economics and Mathematical Systems, Vol. 632 (Springer, Heidelberg, 2009).

[54] Schiavo, S., Reyes, J. and Fagiolo, G., International trade and financial integration: A weighted network analysis (2009), Quant. Finance (forthcoming).

[55] Schweitzer, F., Fagiolo, G., Sornette, D., Vega-Redondo, F., Vespignani, A. and White, D. R., Economic networks: The new challenges, Science 325 (2009) $422-425$.

[56] Serrano, M. A. and Boguñá, M., Topology of the world trade web, Phys. Rev. E 68 (2003) 015101.

[57] Serrano, M. A., Boguñá, M. and Vespignani, A., Patterns of dominant flows in the world trade web, J. Econ. Interact. Coord. 2 (2007) 111-124.

[58] Snijders, T. A., van de Bunt, G. G. and Steglich, C. E., Introduction to stochastic actor-based models for network dynamics, Soc. Netw. (2009).

[59] Sornette, D., Predictability of catastrophic events: Material rupture, earthquakes, turbulence, financial crashes and human birth, Proc. Nat. Acad. Sci. USA 99(Supp 1) (2002) 2522-2529.

[60] Sornette, D., Critical Phenomena in Natural Sciences, Springer Series in Synergetics, 2nd edn. (Springer, Heidelberg, 2006).

[61] Sornette, D., Deschatres, F., Gilbert, T. and Ageon, Y., Endogenous versus exogenous shocks in complex networks: An empirical test using book sale rankings, Phys. Rev. Lett. 93 (2004) 228701. 
[62] Sornette, D. and Woodard, R., Financial bubbles, real estate bubbles, derivative bubbles, and the financial and economic crisis (2009), http://arxiv.org/abs/0905.0220, to appear in the Proceedings of APFA7.

[63] White, D. R. and Johansen, U., Network Analysis and Ethnographic Problems (Lexington Press, Boston, 2005).

[64] Young, H., Individual Strategy and Social Structure: An Evolutionary Theory of Institutions (Princeton University Press, 1998).

[65] Zhao, Z., Calderón, J., Xu, C., Fenn, D., Sornette, D., Crane, R., Hui, P. M. and Johnson, N., Common group dynamic drives modern epidemics across social, financial and biological domains (2009), http://arxiv.org/abs/0907.3600. 